\title{
Processing locational and orientational information
}

\author{
RUTH H. MAKI, WILLIAM S. MAKI, JR., and LINDA G. MARSH \\ North Dakota State University, Fargo, North Dakota 58102
}

\begin{abstract}
In choice reaction time (RT) tasks, college students verified the truth of displays expressing spatial relations between two objects. The relations were locational (A is left of B) or orientational (A and $B$ are horizontal). The objects were names of states in the United States, symbols, or letter arrays. The objects were memorized prior to the display (states and letters) or were presented as part of the display (symbols and letters). In the location tasks with both states and symbols, locatives were spatial (right, left, above, below) or compass (north, south, east, west). Distance between states was also varied. When location was judged, horizontally aligned stimuli resulted in slower responses than vertically aligned stimuli, independently of materials and locative set. Reaction time was inversely related to distance. When orientation was judged, responses to horizontal pairs of states were slower than responses to vertical pairs of states, responses to horizontal pairs of letters were faster than responses to vertical pairs, and RT did not depend upon the orientation of symbols. This pattern of results suggests that orientational judgments are influenced by type of materials and the entext to which the material has been encoded (i.e., memorized). Locational judgments reflect a potent source of difficulty not present in orientation tasks, namely, telling left from right. Alternative explanations of the right-left effect are discussed.
\end{abstract}

Any pair of objects in space possesses three attributes. First, with respect to the observer, the objects have some orientation; for example, two objects A and B might be vertically oriented. Second, within an orientation, one object has a location relative to the other; for example, Object A is above B. Third, the two objects are separated by some distance; in the example, Object $A$ is $5 \mathrm{~cm}$ above Object B. The three attributes seem integral (Garner, 1970 ) in the sense that it is difficult to imagine two objects which lack any one or more of the three attributes. Consequently, ultimate description of processing spatial information must be based on knowledge of how all three attributes influence performance. The present set of experiments aimed to further our understanding of how people process spatial information, by studying the effects of variations in these attributes on performance of choice reaction time (RT) tasks.

Some of the experiments reported here compared the speed of locational discriminations when two objects were arranged vertically (e.g., A is above B) and horizontally (e.g., A is left of B). Corballis and Beale $(1970,1976)$ have marshalled evidence which suggests that making mirror image discriminations is very difficult in bilaterally symmetrical organisms. Such discriminations involve judgments within the horizontal dimension because the observer must determine

These experiments were reviewed in a paper presented at the Psychonomic Society meeting, St. Louis, 1976. L. G. Marsh is now at the University of California, Berkeley. We thank W. Siders and G. Hauge for assistance with the experiments. Send reprint requests to R. H. Maki, Department of Psychology, North Dakota State University, Fargo, North Dakota 58102. whether, for example, a line extends from right to left $(/)$ or from left to right () . A symmetry analysis suggests that discriminations within the horizontal dimension ought to be more difficult than discriminations within the vertical dimension, since there is symmetry within the nervous system with respect to the sagittal plane (right vs. left), but not with respect to the horizontal plane (above vs. below). In support of such an analysis, Rudel and Teuber (1963) had shown that children aged 3-5 years have much more difficulty learning a discrimination in the horizontal dimension $(\subset$ vs. $\supset$ ) than learning a similar discrimination in the vertical dimension $(\cup$ vs. $\cap)$. The acquisition differences largely disappeared by age 8 years, suggesting that the differential difficulty in the two dimensions might be eliminated with age.

With adults, however, there is little evidence that right-left discriminations are more difficult than abovebelow discriminations. In their recent book, The Psychology of Left and Right, Corballis and Beale (1976) state that "it has been claimed that people may take longer to discriminate left-right mirror images than up-down mirror images, but the evidence is not very compelling" $(1976$, p. 36$)$. Some of the evidence which they reviewed did not involve tasks in which right-left discriminations were actually required, and other data could be interpreted in ways which did not involve right-left difficulties. For example, Butler (1964) found that subjects took longer to respond "same" or "different" to two T shapes which were oriented in a left-right direction than to shapes oriented in an up-down direction. However, this may have more 
to do with familiarity with the up orientation (the letter T) than with any left-right difficulty.

There is also evidence that mirror image generalization occurs in recognition of real-world scenes. Standing, Conezio, and Haber (1970) presented a large number of photographs and then tested recognition of them. Subjects were just as likely to recognize a mirror image of the original photograph as they were to recognize the original photograph, and they were generally unable to report whether or not the photograph had been reversed. However, in this task, right-left orientation is relatively unimportant and, hence, subjects may not have attended to it. Another study which suggests that memory for locations within the horizontal dimension might be poorer than memory for locations within the vertical dimension was done by Mandler and Parker (1976). In their study of memory for organized real-world scenes, they presented pictures of objects organized in a scene and later required subjects to place pictures of the objects in their earlier presented locations. Correlations between presented locations and reconstructed locations were higher along the vertical than along the horizontal dimension; memories for horizontal locations seemed to be worse than memories for vertical locations. Mandler and Parker explained this in terms of knowledge abstracted from real-world invariances. Vertical locations in the real world are relatively invariant, but horizontal locations are rarely specified in one's knowledge about the world; roofs are known to be generally above doors and windows, but doors may be to the right or left of windows. Hence, vertical information was more easily reconstructed from general knowledge than was horizontal information.

Other evidence concerning the difficulty of making right-left discriminations comes from speeded tasks in which subjects have been explicitly instructed to discriminate right from left and above from below. However, the conclusions to be drawn from such evidence are not clear cut; some studies show that rightleft judgments are more difficult than above-below judgments for adults, while others suggest that they are not. Harris (1975) showed that college students made more errors when instructed to draw, as fast as possible, a form to the right or left of another form than when instructed to draw the form above or below another. In terms of RT, there are few studies in which speed on the horizontal dimension can be compared with speed on the vertical dimension. Olson and Laxar (1973) required subjects to make right and left judgments about the location of a dot relative to the word "right" or "left." On the average, such judgments took $737 \mathrm{msec}$. They compared their data with those of Clark and Chase (1972) and with those of Seymour (1969), who used similar materials but required abovebelow judgments. Mean RTs in the latter studies were $728 \mathrm{msec}$ and $790 \mathrm{msec}$. It is not apparent from this interexperimental comparison that right-left judgments take longer than above-below judgments. However, Just and Carpenter (1975, Experiment 3) required both above-below and right-left judgments about location of an item within letter arrays and found that, on the average, above-below judgments took less time $(3,302 \mathrm{msec})$ than right-left judgments $(3,976 \mathrm{msec})$; although this difference appears large and consistent in their data, its statistical significance was not established since they did not analyze their data for the effect. One goal of the first two experiments reported below, then, was to determine whether right-left locational judgments result in poorer performance in a RT task than do above-below judgments.

In tasks like those described below, in which locational judgments are called for, knowledge of two attributes must underlie a correct response. Implicit in saying that Object $\mathbf{A}$ is above Object $B$, for example, is the knowledge that Object $A$ and Object $B$ are oriented vertically with respect to some third point in space. Therefore, processing locational information would seem one step more complex than processing orientational information in which knowledge of neither location nor distance need contribute to a correct response. That is, saying that Object $A$ and Object $B$ are oriented vertically demands no information about the specific location of one with respect to the other, nor about the distance separating the two objects. Comparison of the speed of orientational judgments for horizontally and vertically oriented stimuli was the goal of the last three experiments to be reported. If it is found that right-left judgments take longer than above-below judgments in the locational task, but that horizontal judgments do not take longer than vertical judgments in the orientational task, then either the spatial discrimination of right and left or the words "right" and "left" rather than the organization of the stimuli would be implicated in the right-left difficulty.

The role of distance between two objects in space in making discriminations between them has recently received interest. Several investigators (e.g., Moyer, 1973; Moyer \& Bayer, 1976; Potts, 1972) have found that the further apart two objects were on some judged dimension, the faster comparisons between symbols representing those objects could be made. Moyer and Bayer (1976) gave the name "symbolic distance effect" to the inverse relationship between psychological distance and speed of comparison. In some of the present experiments, this variable was manipulated in the context of locational and orientational judgments.

Type of stimulus materials was also varied. Names of pairs of states of the United States were used as natural stimuli stored in memory. Performance on locational and orientational judgment tasks with these stimuli was compared with performance on the same tasks with arbitrary symbols which were presented as perceptual stimuli. Finally, performance on the 
orientational task with arbitrary letter arrays was compared when they were or were not stored in memory.

\section{EXPERIMENTS 1 AND 2}

Locational judgments were required with the names of states of the United States as stimuli. Pairs of these natural stimuli were chosen because they can be varied both in terms of orientation (east-west vs. north-south) and distance (adjacent vs. nonadjacent). The basic question of interest was whether locational judgments would be longer within the horizontal (eastwest) than within the vertical (north-south) dimension. Both compass locatives (east, west, north, south) and spatial locatives (right, left, above, below) were used to insure that any difference observed between locational judgments for horizontally and vertically oriented stimuli were not due to specific words.

However, the natural stimuli allow for some possible confoundings with orientation. For example, the United States map is more extensive horizontally than vertically. This means that horizontally oriented pairs of states were drawn from a dimension with a greater range of possible values than were vertically oriented pairs. Gravetter and Lockhead (1973) have shown that stimulus range affects discrimination performance; equidistant pairs of stimuli tend to become less well discriminated as stimulus range increases. Any differences found between horizontal and vertical discriminations with states might be due to this or some other property of the stimuli and not due to the requirement of telling right from left. Therefore, arbitrary symbols which were present during the judgment task were also used. This allowed for comparison of locational judgments of horizontally and vertically oriented stimuli both when they were natural ones stored in memory and when they were artificial ones perceptually present.

\section{Method}

Materials and design. In Experiment 1, sentences were constructed from 32 pairs of states. In each of 16 "east-west" pairs, the two states were of approximately the same latitude, and in each of the other 16 "north-south" pairs, the states were of approximately the same longitude. Within each set of 16 pairs, one half of the pairs contained states that were adjacent to each other ("near") and the remaining pairs contained states that were nonadjacent ("far"). East-west and north-south nonadjacent states were chosen such that they were about equally far apart. Members of state pairs were used equally often as subjects and in predicates of sentences of the form, "(state) is (locative) (state)." For east-west pairs, locatives were "east of," "west of," "right of," and "left of." For north-south pairs, locatives were "north of," "south of," "above," and "below." Therefore, one half of the sentences involving each pair of states was true and one half was false.

In Experiment 2, names of two symbols were substituted for names of states, and the symbols appeared directly above the stimulus sentences. The symbols were a typed asterisk (referred to as "star") and a lowercase letter o (referred to as "circle"). The star was above or below the circle or left or right of the circle. The form of the sentences and the locatives used was the same as with the state stimuli. Symbol names appeared equally frequently as subjects and objects in sentences.

In Experiment 1 , the order in which compass and spatial locatives were presented varied between subjects. For one half of the subjects, two sessions of reactions to sentences containing compass locatives preceded two sessions of responding to sentences containing spatial locatives; for the remaining subjects, the order was reversed. Four within-subjects factors were manipulated to form a 2 by 2 by 2 by 2 design. These factors were true orientation (horizontal vs. vertical), truth (true vs. false), distance (near vs. far), and locative (compass vs. spatial). Within each of the eight cells of the design defined by true orientation, distance, and truth, 16 sentences were constructed from eight state pairs orthogonally combined with two specific locatives (e.g., above and below). This combination resulted in 128 sentences. Except for the locatives, the same 128 sentences were used in both the spatial and compass conditions. In Experiment 2, this design was repeated with the arbitrary symbols except that distance was not a variable. Since the same number of sentences were presented, the number of sentences per cell in this design was twice that of the design with state stimuli.

Each subject served in four 20-min sessions during each of which three trays of 32 slides were presented. The first tray was regarded as warm-up, and the last two trays provided the data reported below. Tray orders were arranged so that data collected during the last two trays of the two sessions spent on either compass or spatial locatives were based on all four trays. Across subjects, each tray was used as warm-up equally often.

Apparatus. Each sentence was typed and placed in slide mounts (for $35-\mathrm{mm}$ film). The sentence was typed with the subject state or symbol occupying the top row of print, the object state or symbol occupying the bottom row of print, and the verb and locative (e.g., is north of occupying the middle row. Insofar as possible, the three rows were centered both vertically and horizontally on the slide. Each tray of slides contained 32 sentences, (four randomized blocks of 8). The sentences were presented to the subject on a rear-projection screen via Kodak Carousel projectors to which Lafayette tachistoscopic shutters had been attached. Timing was controlled by a LVB Logic Box and RTs were measured to the nearest millisecond with a Hunter Klockounter. The subject was seated about $45 \mathrm{~cm}$ in front of the screen, and stimuli on the screen occupied a space of about $21 \times 15 \mathrm{~cm}$. Two microswitches, mounted about $22 \mathrm{~cm}$ apart on a small console, were used to detect responses.

Procedure. Subjects were instructed to read each statement and decide whether it was true or false based on their knowledge of United States geography (Experiment 1) or on the locations of the symbols at the top of the slide (Experiment 2). True statements were indicated by pressing a switch with the index finger of the preferred hand; false statements were indicated by pressing the other switch with the index finger of the other hand. Each trial began with a warning signal (a cross composed of typed lowercase letter xs) for $.5 \mathrm{sec}$, followed immediately by the sentence. The subject's response terminated display of sentence and stopped the Klockounter. A short break was provided after every 32 slides, during which slide trays were changed.

Subjects. Twelve subjects served in each experiment. The subjects were volunteers from introductory psychology classes at North Dakota State University and received bonus points toward their grades as compensation.

\section{Results}

Experiment 1. Mean RTs were computed for each subject by averaging over 16 or fewer RTs from specific 
Table 1

Mean Reaction Times in Milliseconds (and Percent Errors) in Experiment 1 With State Names as Stimuli for Compass and Spatial Locatives

\begin{tabular}{|c|c|c|c|c|c|}
\hline \multirow[b]{3}{*}{ Truth } & \multirow[b]{3}{*}{ Distance } & \multicolumn{4}{|c|}{ Orientation } \\
\hline & & \multicolumn{2}{|c|}{ Vertical } & \multicolumn{2}{|c|}{ Horizontal } \\
\hline & & Above-Below & North-South & Right-Left & East-West \\
\hline True & $\begin{array}{l}\text { Near } \\
\text { Far }\end{array}$ & $\begin{array}{l}2347(9.9) \\
2175(5.8)\end{array}$ & $\begin{array}{l}2584(8.9) \\
2218(5.2)\end{array}$ & $\begin{array}{l}3186(21.9) \\
2716(6.2)\end{array}$ & $\begin{array}{l}3004(25.5) \\
2622(5.8)\end{array}$ \\
\hline False & $\begin{array}{l}\text { Near } \\
\text { Far }\end{array}$ & $\begin{array}{l}2587(7.8) \\
2344(6.8)\end{array}$ & $\begin{array}{l}2709(8.3) \\
2392(4.7)\end{array}$ & $\begin{array}{l}3435(22.9) \\
3035(9.9)\end{array}$ & $\begin{array}{l}3238(23.9) \\
2750(6.2)\end{array}$ \\
\hline
\end{tabular}

locatives and specific state pairs. RTs resulting from errors (or those in excess of $10 \mathrm{sec}$ ) were not included in the analyses. These data were subjected to an analysis of variance in which the within-subjects factors were true orientation, distance, truth, and type of locative, and the between-subjects factor was ordering of type of locative (spatial first vs. compass first). The findings of primary interest are based on data from the withinsubjects manipulations, and these mean RTs are summarized in Table 1 (averaged over groups). Sentences involving states oriented vertically were verified more quickly than those sentences involving horizontally oriented states $\left[\mathrm{F}(1,10)=39.53\right.$, MSe $=4.07 \times 10^{5} \mathrm{msec}$, $\mathrm{p}<.05$ (the significance level adopted throughout)] . As Table 1 shows, this was done whether the locatives were spatial or compass, although the size of the orientation effect tended to be larger with spatial locatives as indicated by the significant interaction $\left[F(1,10)=5.74, \mathrm{MSe}=1.91 \times 10^{5}\right]$. Nevertheless, the orientation effect was present for both spatial locatives $\left[\mathrm{F}(1,10)=31.71, \mathrm{MSe}=4.03 \times 10^{5}\right]$ and for compass locatives $\quad\left[\mathrm{F}(1,10)=22.53, \quad \mathrm{MSe}=1.95 \times 10^{5}\right]$. Sentences with nonadjacent (far) state pairs produced shorter RTs than did sentences involving near state pairs $\left[\mathrm{F}(1,10)=155.05, \mathrm{MSe}=3.90 \times 10^{4}\right]$. True responses were faster than false responses $[F(1,10)=13.85$, MSe $\left.=1.45 \times 10^{5}\right]$. Finally a significant practice effect was noted as a three-way interaction involving groups of subjects, spatial vs. compass locatives, and distance $\left[\mathrm{F}(1,10)=9.09, \mathrm{MSe}=3.33 \times 10^{4}\right]$. The difference between near and far state pairs was largest for the locatives which were presented first to each group.

Frequencies of errors were subject to the same analysis of variance as outlined above; these data are also summarized in Table 1 as mean percentages. The pattern was the same as for RTs except that distance and orientation interacted $[\mathrm{F}(1,10)=25.69$, $\mathrm{MSe}=2.15$ errors] ; the difference between horizontal and vertical pairs was larger for near than for far state pairs.

Experiment 2. Mean RTs and percent errors as a function of orientation, locative, and truth are presented in Table 2. The analyses paralleled exactly those in Experiment 1, except that the distance variable was not included; therefore, the base cell frequency was 32 . When symbols were oriented vertically, sentence verification was significantly faster than when they were oriented horizontally $[\mathrm{F}(1,10)=18.78$, $\left.\mathrm{MSe}=8.99 \times 10^{4}\right]$. True responses were faster than false responses $\left[\mathrm{F}(1,10)=75.79, \mathrm{MSe}=9.42 \times 10^{3}\right]$. The type of locative (spatial vs. compass) experienced first produced the slowest reactions $[\mathrm{F}(1,10)=8.27$, $\left.\mathrm{MSe}=1.15 \times 10^{5}\right]$. Sentences with compass terms resulted in more errors than did statements with spatial terms $[F(1,10)=5.41, \quad M S e=3.73]$. More errors were committed when symbols were oriented horizontally than when they were oriented vertically $[\mathrm{F}(1,10)=20.07, \mathrm{MSe}=1.20]$.

\section{Discussion}

The finding that near state pairs resulted in longer RTs and more errors than far state pairs is similar to the symbolic distance effects described by others (Moyer, 1973; Moyer \& Bayer, 1976; Potts, 1972). It extends their findings to names of states which can be placed on an actual distance continuum. The farther apart the actual state pairs, the easier it was to make a discrimination using the names of those states.

For both the symbols and states, judgments within the horizontal dimension were more difficult than those within the vertical dimension. When locational judgments were required, the orientation effect was not

Table 2

Mean Reaction Times in Milliseconds (and Percent Errors) in Experiment 2 With Symbols as Stimuli for Compass and Spatial Locatives

\begin{tabular}{cccccc} 
& \multicolumn{3}{c}{ Orientation } \\
\cline { 2 - 5 } & \multicolumn{2}{c}{ Vertical } & \multicolumn{2}{c}{ Horizontal } \\
\cline { 2 - 5 } Truth & Above-Below & North-South & & Right-Left & East-West \\
\hline True & $1341(2.3)$ & $1466(7.8)$ & $1610(8.1)$ & $1702(8.8)$ & $1911(9.6)$ \\
False & $1485(4.2)$ & $1641(8.6)$ & $1772(8.8)$ & \\
\hline
\end{tabular}


material specific, but was found with both natural stimuli in memory and perceptual artifical stimuli. The set of locatives used in these experiments had rather minor effects. With symbols, more errors were made with compass than with spatial terms, perhaps because it is unnatural to think of symbols in terms of the compass. With states, the less natural spatial terms produced larger horizontal-vertical effects on RT, but the orientation effect was present with both sets of terms.

Control experiments. While right-left/east-west judgments were harder than above-below/north-south judgments for both state and symbol stimuli, the results might be explained by factors other than a difficulty in telling right from left. For horizontal stimuli, the hand which was used for the response was incompatible with the correct relationship of the stimuli on half of the trials. For example, if "left of" was the correct relationship, the right-handed subject pushed the right switch to indicate the truth of the statement. Such incompatibility on the horizontal dimension might account for the longer RTs and more errors than on the vertical dimension, where no such incompatibility existed. Experiment 1 showed that compass locatives operate very much like spatial locatives, so the explanation is feasible for the compass as well as for the spatial terms. If the horizontal-vertical effects were simply due to differential compatibility, a response which does not require use of two hands should eliminate the superiority of vertical over horizontal judgments. In this control experiment, states were used as stimuli with compass locatives. The response was a nonspatial vocal one (i.e., saying "true" or "false" in response to the presented sentences).

Materials, design, and procedures were identical to the half of Experiment 1 using compass locatives, except that vocal responses were detected by voice activated relay. Twelve subjects served in the experiment. As in the earlier experiment, horizontally oriented pairs led to significantly slower RTs $(2,522 \mathrm{msec})$ than vertically oriented pairs $(2,097 \mathrm{msec})[\mathrm{F}(1,11)=46.08$, $\mathrm{MSe}=9.41 \times 10^{4} \mathrm{l}$. Therefore, the orientation effect clearly arises from causes other than differential compatibility between stimuli and responses associated with vertical and horizontal dimensions.

Another possible confounding was ruled out in the second control experiment. The sentences in Experiments 1 and 2 were typed with the subject state or symbol on one line and the predicate state or symbol two lines beneath it. Thus, this portion of the display was basically vertical, and perhaps more compatibility with vertically arranged symbols or state names can explain the results. In the second control experiment, symbols ( + and $o$ ) were typed vertically or horizontally at the top of slides and a sentence of the form, " $(+$ or o) (spatial locative) (o or + )" was typed horizontally on one line beneath these. Since the actual symbols and not their names were used, there was actually a physical match for some horizontal stimuli and the subjects and predicates of the sentences. Thus, there was more compatibility for horizontally oriented symbols and the sentence arrangement than for vertically oriented symbols. If the difference between horizontal and vertical stimuli seen earlier was due to stimulus compatibility, then the orientation effect should reverse in this experiment.

The design and procedures were essentially the same as those in the part of Experiment 2 with spatial locatives, except that the voice-activated relay was used to detect the vocal response "true" and "false." Fourteen volunteer subjects participated. Vertically oriented symbols yielded mean RTs of $1,252 \mathrm{msec}$, while horizontally oriented symbols yielded RTs of $1,365 \mathrm{msec}$. This difference was significant $\left[\mathrm{F}(1,13)=28.22, \quad \mathrm{MSe}=6.40 \times 10^{3} 1\right.$. These results make it clear that the superiority of judgments in the vertical as compared to the horizontal dimension observed in the first two experiments was not due to differential compatibility between the sentence configurations and the orientation of the objects.

Neither differential stimulus-response compatibility nor differential stimulus-display compatibility in the horizontal and vertical dimensions can explain the slower discriminations on the horizontal dimension found in Experiments 1 and 2. Making discriminations in the horizontal dimension is more difficult than making discriminations in the vertical dimension. This may be due to some difficulty in perceiving the relative locations in the horizontal dimension or in comprehending the words "right" and "left" or "east" and "west." While the present experiments do show that there is a difficulty in making right-left discriminations with several types of materials, they do not allow an answer to the question of whether it is a perceptual or a verbal difficulty.

\section{EXPERIMENTS 3 AND 4}

The superiority of locational discriminations within the vertical as compared to the horizontal dimension can be explained by a perceptual or verbal difficulty with right and left. One model of processing for the tasks used in Experiments 1 and 2 and the control experiments involves retrieving the general state locations from memory (e.g., Arizona and New Mexico are beside each other in the southwest) or encoding the general pattern of the perceptual stimuli (e.g., side by side or up-down). At this stage, the subject should be able to discriminate whether the two states or stimuli are horizontal or vertical in orientation, although knowledge about specific locations has not yet been retrieved or perceived. The next stage would involve a more precise analysis of the two stimuli to determine specific locations (i.e., which one is above, below, 
left of, or right of the other). During this stage, horizontal discriminations would be more difficult than vertical discriminations as shown in Experiments 1 and 2 and the control experiments. Such a model would predict that determining whether two stimuli are vertical or horizontal would be equally fast for horizontal and vertical configurations, but that locational judgments (i.e., deciding whether a given stimulus is right or left of, above or below another stimulus) would be faster for vertical than for horizontal configurations.

An alternative model of processing involves obligatory retrieval of the exact locations of states or precise encoding of stimulus positions. That is, in order to retrieve the locations of Arizona and New Mexico, it might be necessary to retrieve their relative east-west locations as opposed to simply retrieving the fact that they are side by side. Although it seems unlikely, it might also be necessary to see that an asterisk is left of a circle rather than simply noting they are side by side. In this model, Stage 1 of the first model discussed above is essentially eliminated, and making horizontalvertical discriminations would also result in superior performance in the vertical dimension.

Experiments 3 and 4 involved discriminating whether stimuli were vertical or horizontal. The terms "right," "left," "above," and "below" were replaced by "horizontal" and "vertical." Experiment 3 involved memorized (state) stimuli and Experiment 4 involved perceptually presented stimuli. If the difficulty with the horizontal dimension observed in the earlier experiments was due to the requirement of telling right from left, and if orientation can be retrieved independently of exact locations, then the horizontal dimension should not be more difficult than the vertical dimension in this orientational judgment task.

\section{Method}

Materials and design. In Experiment 3, orientation of states (horizontal vs. vertical), distance (adjacent vs. nonadjacent state pairs), and truth of the relations expressed in the stimulus materials were the main factors manipulated within subjects. Four sets of four states which are adjacent (near) and four sets of four states which are nonadjacent (far) were selected. For example, Arizona, New Mexico, Colorado, and Utah made up one set of near states. Arizona paired with New Mexico created a horizontal pair, while Arizona paired with Utah made up a vertical pair. Within each set of four far states, the distance between vertically oriented pairs was about equal to the distance between horizontally oriented pairs. One member of each pair was typed in the uppermost of two rows of print on a slide so that the first letter was flush with the leftmost margin of the slide. The second member of a pair was typed in the second row so that the last letter was flush with the rightmost margin. Each state name was used in each position equally often. Two lines beneath the second state name was either the word "horizontal" or the word "vertical" centered between the right and left margins.

In Experiment 4, each slide contained a lowercase letter $x$ and a lowercase letter $o$, typed either side by side or one above the other. Each letter appeared in all positions equally often. The word "horizontal" or "vertical" was typed beneath the stimuli. On one half of the slides, the letters appeared "far" apart $(12.85 \mathrm{~cm}$ when projected on the screen), and on the other half they appeared "near" each other $(1.75 \mathrm{~cm}$ when projected).

For both sets of stimuli, four orders (trays) of 32 displays (slides) were formed. Three trays were presented during each of two sessions to every subject, and tray orders were balanced so that each of the four trays appeared as the first, practice tray of each session equally often.

Procedure. Subjects were instructed to decide whether the word "horizontal" or "vertical" at the bottom of each slide accurately described the relationship between the geographic locations of the states named in the upper two rows or whether the word correctly described the orientation of the symbols. True and false responses were indicated by the subject's pressing a switch with the index fingers of the preferred and nonpreferred hands, respectively. Pilot data indicated that the state task was very difficult, so subjects were given immediate, posttrial feedback concerning the correctness of their responses during the practice tray on each day. No feedback was given in the symbol task. Other procedures were the same as those in Experiments 1 and 2.

Subjects and apparatus. For Experiment 3, 16 subjects were recruited from the same pool and rewarded for their services as in the previous experiments. Twelve subjects served in Experiment 4. The apparatus was the same as in the earlier experiments.

\section{Results}

Experiment 3. Both RT and error data are presented in Table 3 in the form of mean RTs and mean percent errors. As indicated earlier, the task proved extremely difficult, and three subjects were dropped (and replaced) because their overall error rates exceeded $40 \%$. Because the error rates were so high, two groups of eight subjects were formed in order to determine whether high-error subjects showed different patterns of results from lowerror subjects. The high-error group consisted of subjects with error rates of between $30 \%$ and $40 \%$, and the lowerror group consisted of subjects with error rates of

Table 3

Mean Reaction Times in Milliseconds (and Percent Errors) in Experiment 3 With States and Experiment 4 With Symbols

\begin{tabular}{|c|c|c|c|c|c|}
\hline \multirow[b]{3}{*}{ Truth } & \multirow[b]{3}{*}{ Distance } & \multicolumn{2}{|c|}{ Experiment 3} & \multicolumn{2}{|c|}{ Experiment 4} \\
\hline & & \multicolumn{2}{|c|}{ Orientation } & \multicolumn{2}{|c|}{ Orientation } \\
\hline & & Vertical & Horizontal & Vertical & Horizontal \\
\hline True & $\begin{array}{l}\text { Near } \\
\text { Far }\end{array}$ & $\begin{array}{l}3204(24.2) \\
3122(22.2)\end{array}$ & $\begin{array}{l}3633(25.4) \\
3254(21.9)\end{array}$ & $\begin{array}{l}871(2.1) \\
879(7.3)\end{array}$ & $\begin{array}{l}846(8.9) \\
875(8.3)\end{array}$ \\
\hline False & $\begin{array}{l}\text { Near } \\
\text { Far }\end{array}$ & $\begin{array}{l}3382(28.1) \\
3468(24.6)\end{array}$ & $\begin{array}{l}3687(28.9) \\
3400(23.8)\end{array}$ & $\begin{array}{r}981(6.2) \\
1067(9.9)\end{array}$ & $\begin{array}{l}991(7.8) \\
996(7.3)\end{array}$ \\
\hline
\end{tabular}


less than $30 \%$. In the following presentation, when orientation effects are described, the terms "horizontal" and "vertical" refer to the actual geographic relationship of the states and not to the word presented on the slide.

Decisions concerning pairs of states oriented vertically were significantly faster than decisions concerning horizontally oriented pairs $[\mathrm{F}(1,14)=5.79$, MSe $\left.=2.14 \times 10^{5}\right]$. Far state pairs produced faster reactions than near pairs $[F(1,14)=6.13$, MSe $=1.38 \times 10^{5} \mathrm{~J}$. However, orientation and distance interactively determined RT $[F(1,14)=15.89$, MSe $\left.=5.5 \times 10^{4}\right]$, because the difference between horizontal and vertical pairs was significant for near states $(3,655$ vs. $3,293 \mathrm{msec}) \quad[\mathrm{F}(1,14)=15.45$, $\mathrm{MSe}=1.36 \times 10^{5} \mathrm{]}$, but not for far pairs $(3,327 \mathrm{vs}$. $3,295 \mathrm{msec})(\mathrm{F}<1)$. True responses were faster than false responses $\left[\mathrm{F}(1,14)=6.99, \mathrm{MSe}=1.54 \times 10^{5}\right]$. None of these results depended on level of accuracy; the between-groups variable (error rate) was not a significant source of variance, nor were any interactions involving that variable significant. Near state pairs produced more errors (26.6\%) than far state pairs $(23.1 \%)[\mathrm{F}(1,14)=5.33$, $\mathrm{MSe}=1.90]$, but orientation had no significant effect on error rates $(F<1)$.

Experiment 4. Mean RTs and average percentages of errors for the symbol task are presented in Table 3. Orientation of the symbols did not reliably influence RT $(F<1)$, but near stimuli produced significantly faster RTs than did far stimuli $[F(1,11)=6.06$, $\left.\mathrm{MSe}=4.03 \times 10^{3}\right]$, and true responses were faster than false responses $\left[\mathrm{F}(1,11)=75.05, \mathrm{MSe}=6.3 \times 10^{3}\right]$. There was a tendency for more errors in the horizontal condition, which suggests some speed-accuracy trading. However, the only significant effect on errors was an interaction between orientation and distance $[\mathrm{F}(1,11)=6.76, \mathrm{MSe}=.56]$. As can be seen in Table 3, the difference between the number of errors for near and far horizontally oriented symbols was very small, but far vertical stimuli produced over twice as many errors as near vertical stimuli.

\section{Discussion}

As in the earlier experiments, near pairs of states yielded longer RTs than far pairs of states. However, with the perceptual stimuli, this distance effect was reversed; near stimuli were responded to faster than far stimuli. With perceptual stimuli, greater physical separation probably necessitated more eye movements, which resulted in longer RTs; with memorized stimuli, physical separation would not require more eye movements, and evidently the orientational judgment task was easier with greater distance between the stimuli. The inverse relationship between distance and RT described by Moyer (1973) appears to hold only for memorized stimuli.
In terms of stimulus orientation, the data do not entirely fit either of the models described in the introduction to Experiments 3 and 4. The first model hypothesized that orientational judgments could be made at a stage prior to that in which left-right effects occur and predicted no horizontal-vertical effect in the orientational judgment task. Support for this model was found with artificial stimuli and with state stimuli in the far condition, since no orientation effects were observed. However, the data obtained with state stimuli in the near condition better fit the second model, in which it was hypothesized that exact locations had to be retrieved or encoded even in orientational judgment tasks. With near state stimuli, horizontal pairs were, indeed, slower than vertical pairs. This suggests that exact locations may have been retrieved only in the near condition, but such a suggestion is inconsistent with the fact that near and far state pairs did not differ in the vertical dimension $(3,293$ vs. $3,295 \mathrm{msec})$. If exact locations were retrieved in the near but not in the far condition, then near pairs should have taken longer than far pairs both for horizontal and for vertical relations. While an explanation for the presence of an orientation effect only for near state pairs is not obvious, it is clear that the pattern of the data from this orientational judgment task was quite different from the pattern observed with the locational judgment task, where horizontal pairs yielded longer RTs than vertical pairs under all conditions.

\section{EXPERIMENT 5}

Two variables were confounded in Experiments 3 and 4: type of materials and whether the stimuli were in memory or present during the response. Experiment 5 was undertaken to determine the effect of this second factor, holding materials constant; orientational discriminations were required when arbitrary letter arrays were either presented perceptually or represented in memory during choice. If the superiority of the vertical dimension with the state stimuli is basic to memorized material, then making horizontal judgments from a memorized letter array should yield slower RTs than making vertical judgments. If, on the other hand, this orientation effect is specific to the organization of materials in memory, no such effect would be expected. Indeed, we might even anticipate a different outcome resulting from storage of arrays consistent with reading habits.

\footnotetext{
Method

Materials and design. Each stimulus display consisted of two parts, an array of four letters configured as $\mathrm{AB}$, and a sentence which referred to the orientation of two letters from that array. Letter arrays were generated randomly with three constraints. No letters were repeated within an array. Except for $Q$ and $I$, all letters were used. Each letter appeared four times, once in
} 
each position within an array. Thus, there were four repetitions of letters $\times 24$ letters $=96$ arrays. Each sentence was one of eight types. The sentence was true (e.g., "A and $B$ are horizontal") or false (e.g., "A and B are vertical"). The sentence referred to one of four pairs of letters which appeared in the array in the top or bottom rows ( $A B$ or $C D$ ) or in the left or right columns (AC or $B D$ ). The arrays were typed in capital letters on the top half of a slide. The sentences were typed in two rows on the bottom half of the slides; the letter pair and verb appeared in the top row, and the words "horizontal" or "vertical" appeared in the bottom row. The apparatus was the same as in previous experiments, except for the addition of a third projector. Twenty-four volunteer subjects were assigned to each of two groups prior to their appearance in the laboratory.

Procedure. Each trial for subjects in the memory group began with a 3-sec presentation of the letter array, followed by a $.5-\mathrm{sec}$ warning signal, and then the sentence. Each trial for subjects in the perception group began with a $3-\mathbf{s e c}$ period of no stimuli, the warning signal, and then both the array and its sentence. Sentences for both groups (and the array for the perception group) remained on the screen until terminated by a response, but for no longer than $7 \mathrm{sec}$. A manual response was used.

\section{Results and Discussion}

Orientation and discrimination. Mean RTs are presented in Table 4 as a function of group, orientation of letter pairs, and truth of sentence. Each mean RT is based on 16 RTs (less errors) for each of 12 subjects. Overall, verification of sentences based on horizontally oriented letter pairs was $435 \mathrm{msec}$ faster than verification of sentences based on vertically oriented pairs $\left[\mathrm{F}(1,22)=23.06, \mathrm{MSe}=1.97 \times 10^{5}\right]$. While subjects in the two groups responded at about the same speed $(F<1)$, the orientation effect was larger for the memory group than for the perception group $\left[\mathrm{F}(1,22)=17.61, \quad \mathrm{MSe}=1.97 \times 10^{5}\right] . \quad$ Horizontally oriented letters were reacted to $815 \mathrm{msec}$ faster than vertically oriented pairs by subjects in the memory group $\left[\mathrm{F}(1,11)=21.70, \mathrm{MSe}=3.67 \times 10^{5}\right]$, but in the perception group the difference was only $55 \mathrm{msec}$, which was not significant $(F<1)$. True responses tended to be faster than false responses $[F(1,22)=13.03$, $\left.\mathrm{MSe}=3.77 \times 10^{4}\right]$, but the size of this effect depended on both group and orientation $[\mathrm{F}(1,22)=6.03$, $\mathrm{MSe}=6.29 \times 10^{4} \mathrm{]}$. For horizontal pairs, both groups responded more rapidly to true than to false sentences, but for vertical pairs, only the perception group showed an advantage of true over false sentences in speed of verification.

Table 4

Mean Reaction Times in Milliseconds (and Percent Error) in Experiment 5 With Letter Arrays as Stimuli

\begin{tabular}{clll}
\multicolumn{2}{c}{ Experiment 5 With Letter Arrays as Stimuli } \\
\cline { 3 - 4 } Truth & Group & \multicolumn{2}{c}{ Orientation } \\
\cline { 3 - 4 } True & Memortical & Horizontal \\
\hline \multirow{3}{*}{ False } & Perception & $2921(16.7)$ & $1931(11.4)$ \\
& Memory & $2858(9.8)$ & $2472(5.2)$ \\
& Perception & $2701(8.3)$ & $2579(6.8)$ \\
\hline
\end{tabular}

Average percent errors are also presented in Table 4 as a function of group, orientation, and truth. Horizontal pairs resulted in fewer errors than did vertical pairs $[F(1,22)=4.43, M S e=1.24]$. True responses tended to be in error more frequently than false responses $[\mathrm{F}(1,22)=5.45, \mathrm{MSe}=.55]$, but differentially so for the two groups $[\mathrm{F}(1,22)=13.75, \mathrm{MSe}=.55]$. In the memory group, true responses were more erroneous than false responses $[F(1,11)=12.44$, $\mathrm{MSe}=.81]$, but in the perception group the difference was smaller and not significant $(F<1)$.

Horizontal letter pairs resulted in faster and more accurate sentence verifications than did vertical pairs. This difference tended to be more pronounced in the memory group than in the perception group. Just and Carpenter (1975), using a locational discrimination task, also found an orientation effect that depended on where the target information was represented. In their Experiment 3, letter arrays were internally represented in memory when the sentences were presented; the rightleft discrimination RTs averaged $674 \mathrm{msec}$ slower than above-below discrimination RTs. In their Experiment 2, sentences were held in memory and letter arrays were externally presented; right-left discrimination RTs averaged only $133 \mathrm{msec}$ slower than above-below discrimination RTs. Just and Carpenter concluded that internal ("mental") representations differed from extemal ("real picture") representations, in that elements of internal representations differ in accessibility but elements of external representations appear equally accessible. The present study supports their conclusion.

Orientational and locational discrimination tasks, however, produce opposing orientational effects. In Just and Carpenter's (1975) experiments with locational discriminations, vertically oriented letter pairs were responded to faster than horizontally oriented pairs. But, in the present experiment with orientational discriminations, the reverse pattern was obtained. It might be argued that the different tasks encourage different encodings, but further examination of the present data renders this possibility unlikely. Just and Carpenter reported that letter pairs from top rows produced faster RTs than those from bottom rows, and that letter pairs from left columns produced faster RTs than letter pairs from right columns. This pattern, they suggested, is consistent with encoding according to reading habits (top to bottom, left to right). The following analyses demonstrate similar effects in the present data.

Row and column effects. RTs based on horizontally oriented letter pairs were examined as a function of group, truth, and the row in which the pair was presented (top vs. bottom). Sentences referring to letters occupying top rows were judged faster than sentences referring to letters occupying bottom rows $(2,179$ vs. $2,418 \mathrm{msec}) \quad\left[\mathrm{F}(1,22)=17.12, \quad \mathrm{MSe}=8.04 \times 10^{4}\right]$. 
Groups did not differ in mean RT $[F(1,22)=3.86$, MSe $\left.=1.19 \times 10^{6}\right]$, but the row effect differed between groups $\left[\mathrm{F}(1,22)=8.49, \mathrm{MSe}=8.04 \times 10^{4}\right]$. In the memory group, top-row pairs were reacted to faster than bottom-row pairs $(1,876$ vs. $2,284 \mathrm{msec})$ $\left[\mathrm{F}(1,11)=19.86, \mathrm{MSe}=1.01 \times 10^{5}\right]$. In the perception group, however, top-row RTs averaged $2,481 \mathrm{msec}$ and bottom-row RTs averaged $2,553 \mathrm{msec}$, and this difference was not significant $(F<1)$. Errors did not vary as a function of row.

RTs based on vertically oriented letter pairs were examined as a function of truth, group, and column in which the pair was presented (left vs. right). On the average, sentences referring to letters from left columns were responded to $138 \mathrm{msec}$ faster than sentences referring to letters from right columns $[F(1,22)=5.21$, $\left.\mathrm{MSe}=8.69 \times 10^{4}\right]$. Once again, groups did not significantly differ in mean $\operatorname{RT}[F(1,22)=3.23$, $\mathrm{MSe}=8.42 \times 10^{5} \mathrm{~J}$, but the size of the column effect varied between groups $[F(1,22)=7.60$, $\left.\mathrm{MSe}=8.69 \times 10^{4}\right]$. For the memory group, left-column sentences were responded to faster than right-column sentences $(2,760$ vs. $3,063 \mathrm{msec}) \quad[\mathrm{F}(1,11)=7.54$, $\left.\mathrm{MSe}=1.46 \times 10^{5}\right]$, but for the perception group the difference was not significant $(2,589$ vs. $2,560 \mathrm{msec})$ $(\mathrm{F}<1)$. Errors were more likely when the letters came from the right $(15.2 \%)$ than from the left $(6.2 \%)$ column $[\mathrm{F}(1,22)=23.46, \mathrm{MSe}=.51]$.

With respect to row and column effects, the present results parallel closely those of Just and Carpenter (1975). In both studies, letters from top rows produced faster RTs than letters from bottom rows, and letters from left columns produced faster RTs than letters from right columns. Therefore, it appears that letter arrays were encoded consistently with reading habits in both studies. Also common to the two studies was the dependence of both row and column effects on the locus of the target information. When letters were represented internally, the effects were quite evident, but when presented externally, the effects virtually disappeared. Given these similarities, it is unlikely that the different tasks (locational vs. orientational discriminations) resulted in different encodings, which in turn were responsible for opposite orientational effects.

If different encodings are not produced by the locational and orientational discrimination tasks, then why is the orientation effect reversed between this experiment and that of Just and Carpenter? The conclusion to be later pursued holds that the locational discrimination task presents a difficulty not found in the orientational discrimination task. In the latter task, subjects discriminate between things based on their locations along horizontal and vertical continua. This means that subjects are faced with "telling left from right" only in locational discriminations. This source of difficulty is so potent as to mostly overshadow the patterns of results obtained in orientational discrimination tasks.

\section{GENERAL DISCUSSION}

In this paper we have documented two main facts. First, when dealing with locational information, adult humans find horizontal orientations more difficult than vertical orientations. Second, when orientational judgments are made, the difficulty of judging horizontally as compared to vertically oriented objects depends upon what the objects are and where the objects are represented.

In Experiments 3 and 5, subjects judged the truth of displays expressing orientational relations between two stimuli. When the stimuli to be judged were presented in the display, there was little difference between times required to make horizontal and vertical judgments. However, when the stimuli were represented in memory, material-specific orientational effects were noted; horizontal judgments were faster for letter arrays, while vertical judgments tended to be faster for state pairs. One variable, then, which determines whether orientational judgments are more difficult for vertical or horizontal stimuli is degree of encoding. Stimuli which are present during the judgment time require little encoding and show no horizontal-vertical difference, but stimuli which have been placed in memory show horizontal-vertical effects with the specific pattern of difficulty depending upon the type of materials.

The second variable which influences orientational judgments is the nature of the materials which are encoded. The results of both the present Experiment 5 and Just and Carpenter's (1975) Experiment 3 suggest that letter arrays are encoded consistently with reading habits; in both experiments, items in top rows were more accessible than items in bottom rows. Because reading proceeds row by row (as opposed to column by column), horizontally oriented letters in Experiment 5 were more rapidly judged than were vertically oriented letters. Therefore, we might say that the organization of letter arrays was primarily horizontal. In contrast, the primary organization of the United States in memory appeared to be vertical. In Experiment 3, vertical pairs of near states were more rapidly judged than horizontal near pairs. Our intuitions suggested the reasonableness of this conclusion (i.e., one tends to name compass points as "north, south, east, west," perhaps since real maps tend to be oriented with respect to north). More objective support was provided by 20 undergraduates from the same pool as those who served in Experiments 1-5. They were asked to "write the names of the four major compass points." Of those subjects, 17 wrote the names in the order listed above, two wrote "north, east, south, west," and one subject wrote "north, south, west, east." While there was some variability with respect to specific ordering, north was always given first.

In addition to showing how orientational judgments are influenced by the encoding of different materials, 
the present data reflect the operation of a variable which distinguishes between orientational and locational judgments. In Experiments 1 and 2, subjects judged the truth of sentences referring to relations between locations of two states or symbols. Relations between states were assumed to be in long-term memory, and relations between symbols were presented via the displays. Regardless of locus of representation (memory vs. perception), horizontally oriented pairs were responded to more slowly than vertically oriented pairs. While we did not require locational judgments of artificial stimuli in memory, Just and Carpenter (1975, Experiments 2 and 3) did, and their data showed that horizontal locational judgments took longer than vertical locational judgments, regardless of materials and degree of encoding. One explanation of the pervasiveness of the slower responding to horizontal pairs may be that locational judgments present subjects with the task of telling left from right.

When two horizontally oriented items must be evaluated with respect to their relative locations, the subject is deciding whether "A is (left/right) of B." In contrast, deciding whether " $A$ and $B$ are horizontal" demands no knowledge of the relative location of $A$ with respect to $B$. The right-left problem is so pronounced that it largely obscures the effects of variations in materials and degree of encoding in the locational task. Citing the left-right problem may account for the different pattern of results between Experiments 1 and 2 (with the locational task) and Experiments 3.5 (with the orientational task), but why should there be a right-left problem at all?

There have been generally two answers to the question raised in other contexts. First, it might be that problems in telling left from right arise from the organism's "hardware." Corballis and Beale (1970, 1976) argued that bilaterally symmetrical creatures will experience difficulty in discriminating right from left to the extent of their symmetry. Second, it might be that such problems arise from the organism's "software." Mandler and Parker (1976) noted that real-world objects have more invariant vertical locations than horizontal locations. That is, a chair might be to the right or left of a wall clock, but rarely do we encounter a chair located above a clock. They proposed that subjects have learned "schemata" for dealing with vertical relations, but not for the more variable horizontal relations. This is why, Mandler and Parker claimed, their subjects remembered vertical locations of objects originally presented in real-world scenes better than horizontal locations. Mandler and Parker's hypothesis does not seem well suited to account for the present findings. While subjects may be more familiar with north-south state pairs than with eastwest state pairs, it is implausible that vertically oriented symbols are more familiar than horizontally oriented symbols. There is nothing in the real world which mandates circles to be generally above stars or vice versa. While subjects do come to the laboratory with schemata called reading habits, this prior knowledge should favor horizontal stimuli, as it appears to have done when orientational judgments were called for in letter arrays. However, Just and Carpenter (1975) found that vertical judgments of letters were faster when locational judgments were required.

While extrapolations from Mandler and Parker's (1976) hypothesis cannot account for our findings, other "software" hypotheses remain viable. Note that the uncertainty in locations along the horizontal dimension is reflected in language. We have many terms available to describe two objects horizontally aligned ("next to," "beside," "side by side"), none of which express location. As far as we know, however, no such words exist which express such spatial ambiguities along the vertical dimension. It may be that encoding of the words "right" and "left" (or "east" and "west") captures some of this uncertainty, and thus the internal representation of such words is imprecisely or slowly constructed. We await future research to resolve the question of the basis for the difficulty experienced by human subjects in telling right from left.

\section{REFERENCES}

Butler, J. Visual discrimination of shape by humans. Quarterly Journal of Experimental Psychology, 1964. 16, 272-276.

Clark. H. H., \& Chase. W. G. On the process of comparing sentences against pictures. Cognitive Psychology, 1972, 3. 472-517.

Corballis, M. C., \& Beale. I. L. Bilateral symmetry and behavior. Psychological Review. 1970, 77, 451-464.

Corballis. M. C., \& BEAlE. I. L. The psychology of left and right. Hillsdale. N.J: Lawrence Erlbaum, 1976.

GARNER. W. $R$. The stimulus in information processing. American Psychologist, 1970, 25, 350-358.

Gravetter. F., \& Lockmead, G. R. Criterial range as a frame of reference for stimulus judgment. Psychological Revien: 1973, 80, 203-216.

Harris, L. J. Spatial direction and grammatical form of instructions affect the solution of spatial problems. Memory \& Cognition, 1975, 3, 329-334.

Just. M. A., \& Carpenter, P. A. The semantics of locative information in pictures and mental images. British Journal of Psychology. 1975, 66, 427-441.

Mander, J. M.. \& Parker, R. E. Memory for descriptive and spatial information in complex pictures. Journal of Experimental Psychology: Human Learning and Memory, 1976. 2. 38-48.

MOYER, R. S. Comparing objects in memory: Evidence suggesting an internal psychophysics. Perception \& Psychophysics, 1973, 13, 180-184.

Moyer, R. S., \& Bayer, R. H. Mental comparison and the symbolic distance effect. Cognitive Psychology, 1976, 8. 228-246.

Olson, G. M., \& Laxar. K. Asymmetries in processing the terms "right" and "left." Journal of Experimental Psychology, 1973, 100, 284-290.

Potrs, G. R. Information processing strategies used in the 
encoding of linear orderings. Journal of Verbal Learning and Verbal Behavior, 1972, 11, 727-740.

Rudel, R., \& Teuber. H.-L. Discrimination of direction of line in children. Journal of Comparative and Physiological Psychology, 1963. 56. 892-898.

Seymour, P. H. K. Response latencies in judgments of spatial location. British Joumal of Psychology, 1969, 60. 31-39.
Standing, L. Conezio, J.. \& Haber, R. N. Perception and memory for pictures: Single trial learning of 2500 visual stimuli. Psychonomic Science, 1970, 19. 73-74.

(Received for publication March 10, 1977; revision accepted June 24, 1977.) 